Migraine, known for centuries to medicine, is a paroxysmal and functional disorder. It is of considerable importance to the physician, and especially to the neurologist, for at least in the latter's out-patient department it is probably the commonest disorder to be seen next to the pure neuroses. In addition it is a cause of much unnecessary anxiety and much may be done for its relief.

\section{Aetiology}

Long before any attempt was made at scientific proof of it, the theory was held that the symptoms of the aura were best explained as due to transitory anoxia of one or more parts of the nervous sytem as the result of narrowing of an appropriate artery, the narrowing to be short-lived and reversible, therefore presumably due to contraction of the blood vessel wall. Although direct proof of this happening is still to be found, there are a number of suggestive pieces of indirect evidence. Arteries to the brain have a muscular coat with which to contract and they have been seen to narrow, by direct observation, though admittedly under abnormal conditions at craniotomy. Vasodilator drugs have repeatedly and immediately abolished the symptoms thought to be due to such constriction, especially the defects in the visual field that are so common in migraine. Electroencephalographic (E.E.G.) changes have been found in the shape of abnormal waves issuing from that portion of the brain appropriate to the field defect, for instance from the opposite occipital lobe during the time that an homonymous hemianopia was being experienced by a patient in an attack of migraine. These E.E.G. changes are consistent with an area of relative ischaemia of the brain. Further work, notably by Schumacher and Wolff (194I) and Wolff (1948) in the United States, has shown that the headache of migraine probably arises from undue dilatation of extracerebral and particularly extracranial blood vessels. It is a common observation that digital pressure upon a superficial temporal artery will abate or abolish the ipsilateral headache of migraine and will continue to do so whilst the pressure is held. It is difficult to imagine that this simple manoeuvre works in any other way than lessening the blood flow through the artery and so relieving a temporary state of undue and painful distensibility of the vessel wall. It is certain that these extracranial arteries are sensible to pain. In some subjects of migraine the superficial arteries of the head may be seen to be unduly dilated during the painful stage of an attack, and indeed only on the side of a unilateral headache. The beneficial effect of ergotamine tartrate on the headache of migraine has also been taken as evidence of its cause, for this drug is a powerful vaso-constrictor. It may be that many or all intracranial and extracranial vessels share in this constriction followed later by abnormal dilatation, but that because of differences in the oxygene demand, between some tissues and others, and differences in the pain sensitivity to undue dilatation from one artery to another there is a variability of effect in terms of symptoms. That some symptoms of migraine, thought to be due to cerebral vaso-constriction, are common and that others, theoretically possible, are rare or do not occur may be due to a varying vulnerability to anoxia of different parts of the brain. Perhaps it is, however, that a transitory disturbance of function of some parts of the brain is more obvious than others to the patient; particularly those concerned with vision.

However much we may pretend a knowledge of the immediate cause of the symptoms, we must admit to considerable ignorance as to what initiates the series of events. A glance at the section given to the aetiology of migraine in most textbooks will reveal this ignorance, for every theoretical possibility is covered by a handful of volumes. The most important predisposing factors are an inherited tendency to this particular set of symptoms together with an inborn ability to worry more than the usual run of folk. Most of the other factors claimed, such as an endocrine one, are probably subservient to those two. It is true that a majority of those who attend an out-patient department are women, but that is not to say that the disorder is less common in males. Occasionally a sufferer is able to convince one that some, most, or more rarely all of his attacks are fired off by a particular 
foodstuff or contact with an allergen of another kind. These are, however, the rarer and lucky ones for they have their treatment within their own grasp and, for this reason, the better ones are not indeed patients. The fact that allergic disorders such as asthma are commoner amongst the relatives of migraine sufferers than others is not a perfect reason to link directly the two complaints, for a predisposition common to both may exist, the kinship being no closer than that. The menopause, a common time for the attacks to ripen, is as much a time for commotion of the patient's mind as it is for her body. Despite the fact that most sufferers have their first attack at the time of puberty it is by no means unknown in earlier years when it may be termed cyclical vomiting, and I think one would rather choose a young team of potential migraine subjects from a study of the children's inheritance and disposition than from their sex or possible endocrine irregularities. Migraine may be diagnosed correctly for the first time at any age after a proper history from the patient is possible, though it is distinctly uncommon for fullblown and classical attacks to begin late in life without a previous history of paroxysmal headaches of the kind that are now associated with other symptoms. Some have stated that migraine and epilepsy are related more than by chance; this is not proven one way or the other but it is not difficult to imagine, if our concept of the immediate cause is true, how a convulsion or other epileptiform happening might arise from cortical anoxia. Every now and again one will see a patient who describes an occasional typical migraine attack culminating in an epileptic convulsion. Oddly enough this occurs some time after the headache has been present, often an unusually severe one, and even longer after the aura when we would suppose the cerebral cortex to be in its most excitable state. Digestive and bowel disturbances are a common association of an attack of migraine and are probably more accurately so described than as a possible aetiological factor. I have seen a few patients in whom the beginning and end of a severe exacerbation of attacks of classical migraine with aura (quite distinctive from the pain of frontal sinusitis) were so closely related to the onset and surgical relief of frank purulent paranasal sinusitis that it was difficult to believe that no relationship existed. This is not to say that sinusitis is a cause of migraine.

There are, too, the interesting examples of apparently reflex initiation of an attack in a susceptible person. For instance an attack may be brought on by watching the cinema screen, particularly after leaving the theatre in bright sunlight. Staring into a thick mist will often do the same thing, particularly if it be illuminated by that intense white light which seems to show up the very moving particles themselves. In both these examples the visualized image and its reflection as a migrainous aura are much alike, and it is as if by seeing such a representation of its own recurring hallucination that a time honoured series of disordered actions are set in motion.

Whether or not a particular psychological disposition may cause a tendency to migraine there is no doubt that anxiety of one kind and another is the commonest reason for exacerbations of frequency and intensity of attacks. A mere change of routine is sufficient in some to cause an attack, hence the 'week-end' or 'Sunday morning' headache not uncommon amongst migrainous subjects.

\section{Pathology}

There is no known macroscopic or microscopic pathology of migraine. In the present state of our knowledge it must be regarded as a functional disorder, in the proper sense of that word, and indeed it seems unlikely that an anatomical lesion of any kind will ever be found for a disorder that has all the qualities of an evanescent change in the body's nervous or biochemical balance. The gross lesions that have been found post-mortem are in those whose intracranial tumour, aneurysm or arteriovenous malformation has been mimicking mis graine, perhaps very closely, for a number of years.

\section{Clinical Picture}

There may be warning symptoms of an approaching attack, perhaps for hours before, in the shape of a mild malaise or fatigue or a vague abdominal upset. Sometimes even there is a sense of unusual well-being. The pattern of such a prodrome tends to be constant for the individual and so he is forewarned. In the classical variety there then comes an aura which is nearly always sensory. Most commonly visual, it may take the form of positive or negative phenomena, the former, properly called hallucinations, may appear as black, white or coloured dots or shapes in the central or peripheral field of vision gradually gaining in intensity and may be spreading so as to ablate a greater or lesser portion of it. These moving, positive images may give way to negative defects in the form of scotomas or partial or complete hemianopias. The classic, scintillating and castellated strip of light, the so-called fortification spectrum or teichopsia, is not by any means the commonest of such phenomena. The spread may be from periphery to centre, or vice versa, and has usually come to full fruition and is slowly fading by the end of 10,20 or 30 minutes. There may be mixtures of these field defects and positive hallucinations in the same patient in the same attack, 
the phenomena may vary from one time to another or an individual may have precisely the same aura in each attack for a lifetime. True diplopia may occur or mere blurring of vision as an aura.

Other sensory aura occur, either by themselves or in addition to the visual ones. Paraesthesiae of varying kinds are the commonest, most often in one or other hand, occasionally both, or around the mouth, lips or tongue, when they are usually bilateral. Again, they are evanescent and may be accompanied by vague clumsiness, awkwardness or weakness of the part affected. A transitory upset of speech at a high functional level, a dysphasia, or more rarely aphasia, may replace or accompany any of these happenings. A severe or indeed complete hemiplegia or monoplegia is an occasional recurring, shortlived aura of migraine, and this has been described, more than once, as a familial happening. A mild or indeed brisk vertigo occurs in some in the period immediately before the headache; it would be of great interest to know the central locus of this particular aura.

In the classical attack, as the aura fades it is replaced by the headache. This usually begins over one frontal region or temple and may remain there or spread to cover most, if not the whole, of one side of the head. Sometimes the ache is bilateral, sometimes mainly or only occipital. Many adjectives will be used to describe the pain; aching, boring or throbbing, but it is the last that may be applied almost universally, particularly as the headache reaches its height. It tends gradually to increase in intensity, commonly at its worst in 30 or 60 minutes from its onset and at that time often associated with nausea and vomiting, which may be repeated. At this stage, too, there is a marked tendency for it to be made worse by change of posture or exertion, the simple act of walking sometimes being intolerable. In the average case it lasts a matter of hours, commonly until lost in sleep, in some a shorter time and, in the few, it may linger for days. The severity of the pain seems to bear no relationship to its length of stay: the most acute may be the shortest. There is a wide variability of the severity of the pain, not only from one patient to the other but from time to time, and it is particularly important to diagnosis to understand that a typical aura may not be followed by a headache and indeed a sufferer from classical attacks may have many, perhaps most, of his headaches without an aura. It is not every patient who will admit to his headaches following his aura in orderly sequence and many insist that these symptoms, thought to be cerebral in origin, are observed during and not before the pain, and may indeed recur once or more in an attack. Many people, sufferers from recurring mild or severe headaches, may wait years for the correct diagnosis until a dramatic aura frightens them to their physician. At the worst the headache is intensely severe and quite disabling, apart from the possible associated vomiting, and the miserable subject may have to take to his darkened room and hope for the respite that comes with sleep. Next day, pain-free and refreshed, he may be aware of vague abdominal symptoms, slight looseness of the bowel or a considerable polyuria lasting a few hours. Observable vasomotor changes may occur at the height of the attack, with or without vomiting, as pallor or flushing of the face or extremities. The heightened colour may be unilateral in the face and indeed only on the side of the pain; it may include the conjunctivae.

In the average susceptible person, such classical attacks, with minor variances, may happen at intervals of weeks or months, perhaps interspersed by an occasional mild affair without an aura. With occasional exacerbations when a crop of attacks may come within days or weeks, they come and go for years, as a rule lessening as middle age is passed and tending, on occasion, to fresh activity for a year or two around the menopause. Their natural history, however, is subject to wide variation and no rule of conduct for migraine can certainly be laid down, unless it is to say that mental tension and anxiety are almost sure to reap a crop.

\section{Prognosis}

Migraine does not, of itself, shorten life nor does it ever lead to death in an attack. Permanent defects of any kind are so rare as to be curiosities, and one wonders whether idiopathic migraine is ever the cause of the occasional lasting hemianopia or other visual field defect that has occasionally been ascribed to it. Admittedly one may conjecture on the possible permanent sequelae of vascular spasm associated with arterial disease, but it must remain a surmise, and that it happens rarely at the most may confidently be stated.

\section{Diagnosis}

The first step is to be familiar with the clinical picture as described by the patient, not only of the classical attack but of its many minor variations. Having so recognized the picture and estimated the true length of its history a careful examination is essential, for two reasons. First and foremost to assure oneself that there are no abnormal physical signs that could be considered relevant to the symptoms, for there are no such abnormal signs in idiopathic migraine. Second, so as to put oneself into a strong position to assuage the patient's anxiety, for nothing is so reassuring as assurance. In the examination an intelligent neurological 
appraisal is the essential, for the conditions that closely simulate migraine are all intracranial ones. Perhaps most important in this examination are the following: Ausculation of the head, the state of the homonymous visual fields as done by confrontation, the optic fundi, the pupils, the ocular movements and the reflexes.

Intracranial neoplasms, primary and secondary, intracranial angiomata and aneurysms are all capable of simulating migraine. Tumours of the occipital lobe by causing visual hallucinations, headache and vomiting are probably the most likely, though not most common, to cause confusion. Almost certainly there will, however, be abnormal signs recognized by attention to those parts of the neurological examinal just referred to. Cerebral angiomata are by no means always associated with abnormal signs, even on the most careful examination, and they tend to mimic migrainous symptoms closely, indeed it is only in later years and with the help of cerebral angiography that their true frequency is being appreciated. Persistently one and the same sided headaches, pulsatile or continuous head noises, the same noise heard as an auscultated bruit, prolonged.visual field defects or the finding of even minimal pyramidal or sensory signs may assist to send the patient for the proper neurological investigation. Intracranial aneurysms, particularly those situated on or near the anterior part of the circle of Willis, may produce recurring headaches, usually frontal and circumorbital and again always on the same side. This one and the same sidedness of such pain, over many attacks, should always arouse the suspicion that the cause is not idiopathic migraine, though this suspicion is not of course inevitably correct. Aneurysms, apart from causing the clinical picture of subarachnoid haemorrhage which migraine does not, may cause transient pareses of the pupil or of the external ocular muscles and the levator of the upper lid. It is probably such aneurysms that have led in the past to the use of the term ophthalmoplegic migraine. It is wise and almost certainly correct to take the view that true migraine is never accompanied by demonstrable paralysis of the eye muscles. The pain of tic douloureux (trigeminal neuralgia) is quite unlike that of migraine in its age of onset, its location, its temporal characters and its qualities; its closest affinity to migraine is in the absence of abnormal neurological signs. The most potent non-neurological cause of misdiagnosis is probably frontal sinusitis which has previously been mentioned as an occasional true exacerbator of migraine. A careful history, however, usually makes plain the differences in quality of the two pains, and perhaps most important their different temporal qualities. In addition, of course, there are the clinical and radiographic signs of sinus infection.

The diagnosis, therefore, is made by an informed listener, who is mindful of the vagaries of migraine, who is alert to other possible causes of a like story but who knows, too, that common things commonly occur, and who has searched for and excluded evidence of a gross intracranial lesion. The need then for special methods of investigation will be occasional. A radiograph of the skull has the merit of helping to reassure the patient; it has, however, little else to offer as an isolated investigation in such cases, for it never shows migraine and comparatively rarely evidence of any of the differential diagnoses. If sufficient doubt of the diagnosis be felt after the clinical examination, it is probably better to refer the patient for possible special neurological investigations.

\section{Treatment}

Many patients, and particularly those who have recently suffered their first aura or who have had a brisk exacerbation of a previously mild and occasional attack, will be in need of reassurance. Such reassurance is an essential part of the treatment for it commonly breaks into the one arc in a vicious circle of worry that is amenable to the doctor. It is likely that this part of such a circle is a small one but of particular importance, for the remainder, though capable of being discussed by the physician, is not often truly resolved by him? As has been said here before, this reassurance, to be effective, should be based upon the doctor's own assurance. Sufficient time and emphasis must be given to it, for it is more important than any medicinal treatment and enhances the subsequent response to drugs. In the average case the use of a simple sedative such as phenobarbital, $\frac{1}{2}$ gr. three or four times a day, or amytal, $\frac{3}{4}$ gr. three times a day, continued for several weeks, will be sufficient to reduce or even temporarily abolish the attacks. Experience shows that such a regime is superior, for the great majority, to an attack upon each headache as it arises. In fact it is better to make an initial attempt at abolishing attacks than curing them as they happen. The majority of sufferers reach the consultant during an exacerbation, years after the migraine became installed, and it is along these lines that he will get his best results. Despite such treatment, there are some whose attacks are sufficiently severe and frequent to warrant a direct assault upon each one, and that is particularly true of the busy professional or business man who does not find it easy to retire to bed with analgesic drugs and await the natural end of an attack. For them, ergotamine tartrate or a related drug is useful. Ergotamine usually has a specific effect on the headache, little or none on 
the aura, and is said to work by virtue of constricting the unduly lax and therefore painful blood vessel. The essence of its use is that it should be given as early in the attack as possible and preferably during the prodrome or at least the stage of the aura. A dose of $2 \mathrm{mg}$. is taken initially by allowing it to dissolve under the tongue and this dose may be repeated in an hour or more if necessary. Perhaps more effective is a subcutaneous injection of a $\frac{1}{2} \mathrm{mg}$., best given by the patient himself, of course, and reserved for the few whose attacks are tending to interfere with their professional lives. This, too, may be repeated. Ergotamine may also be used prophylactically but it is contraindicated in pregnancy or with advanced arterio-sclerosis. It is not without unwanted side effects, particularly nausea, and this may interfere with an otherwise excellent therapeutic result. Dihydroergotamine is said to have fewer toxic effects with an equal therapeutic one and may be given parenterally in doses of $\mathrm{I}$ to $2 \mathrm{mg}$. It would not be useful to make a list of all the analgesic drugs that have been used for the relief of the headache of migraine. Probably the most effective is codeine phosphate in $\frac{1}{2}$ to 2 gr. doses or mixtures containing it, but of course individual preferences may dictate the use of other drugs.
It is seldom that continued sedation, analgesic drugs or ergotamine tartrate (femergin) either separately or in one or other combination, fail to produce a good effect. Several pages could be given over to a discussion of the relative merits of a hundred treatments for migraine. This alone should convince the doctor that not one of them is the specific for all his patients. As with many other disorders, particularly functional ones, it is hard not to believe that some of the suggested treatments are merely vehicles for an infectious enthusiasm. Which ever method is used, however, there is no alternative to a reassuring discussion with the patient, an explanation of his complaint and an attempt made to reorientate him to the troubles that he may have. It is a mistake to hurry to vasoconstrictor drugs without at first trying simpler remedies and especially those that reach further back than the immediate 'mechanical' cause. Time taken at the first interview, possibly ill-afforded, will repay itself a hundredfold in the future.

\section{REFERENCES}

SCHUMACHER, G, and WOLFF, H. G, (I94I) 'Experimental Studies in Headache,' Arch. Neuron. and Psychiat., xiv, 199. WOLFF, H. G., (1948) 'Headache and Other Head Pain.' London, Oxford University Press.

\title{
THE PRESENT POSITION IN THE TREATMENT OF CHRONIC ULCERATIVE COLITIS
}

\author{
By Rupert S. CoRbetT, M.Chir., F.R.C.S. \\ Surgeon, St. Bartholomew's Hospital, London
}

Chronic ulcerative colitis has been talked about more in recent years than ever before. It is best qualified by adding the term 'non-specific' as in this way the dysenteries are definitely ruled out. It is of the greatest importance to do this as amoebic dysentery can easily similate this disease. Although the dysenteries are of rare occurrence in this country, patients did present themselves after the war years as a result of service in the East.

The disease is a serious one and unfortunately the cause is still unknown. It is serious because when once a patient is a victim he never seems to recover completely. On the other hand, a high proportion can continue to live without severe handicap on medical treatment alone and this may have to be resorted to only at intervals. This emphasizes the characteristic nature of the disease in that it is very liable to intermissions and there may be quite long periods of remission of symptoms. The number of cases that call for surgical treatment is probably not more than 25 per cent. When this form of treatment is undertaken it always means an ileostomy-temporary or permanent. Looking back on the other forms of surgical treatment, appendicostomy was very popular. It was a minor operation and allowed the application of medication to the colon, which was thought to be of value. Sir Arthur Hurst showed, however, that if one were to rely upon the introduction of antiseptics and astringents they could 